\title{
Performance study of IEEE 802.16d under Stanford University Interim (SUI)Channel
}

\author{
Abdul HashibSiddique ${ }^{1}$, A K M Arifuzzman ${ }^{2}$ and Mohammed Tarique ${ }^{3}$ \\ ${ }^{1}$ Department of Electrical Engineering, The Petroleum Institute, Abu Dhabi, UAE \\ absiddiqueepi.ac.ae \\ ${ }^{2}$ Department of EEE, American International University-Bangladesh, Dhaka, Bangladesh \\ arifuzzamaneaiub.edu \\ ${ }^{3}$ Department of Electrical Engineering, Ajman University of Science and Technology, \\ Fujairah, UAE \\ m.tariquedajman.ac.ae
}

\begin{abstract}
Wireless communications are subject to three major constraints: (i) a complex and unpredictable fading channel, (ii) a scarce radio spectrum, and (iii) limitations on the power and the size of handheld terminals. New technologies have been introduced and evolved based on these constraints. Among all of these technologies Worldwide Interoperability for Microwave Access (WiMax) has drawn considerable attention for broadband wireless communication. The WiMax technology has been standardized under IEEE 802.16d and Orthogonal Frequency Division Multiplexing (OFDM) is the core of the WiMaxtechnology.In order to investigate the performances of WiMax system a practical channel model is required. Many channel models have been proposed to mimic a real world scenario including Stanford University Interim (SUI) channel. This model has attracted considerable attentions of the researchers in the last few years. In this paper, the performances in terms of bit error rate (BER) of IEEE 802.16d have been investigated by using different SUI channel model. Some popular digital modulations like BPSK and QPSK have been considered. The simulation results have been presented and compared for different SUI channel models.
\end{abstract}

\section{KEYWORDS}

WiMax, IEEE 802.16d, OFDM, Cyclic Prefix, BPSK, QPSK.

\section{INTRODUCTION}

In the recent years there has been a rapid growth in the Broadband Wireless Access (BWA) networks. BWA is constantly acquiring popularity as an alternative of "last-mile" technology instead of Digital Subscriber Line (DSL) and cable modems technologies.Because of its wireless nature it is faster to deploy, easier to scale and more flexible. Therefore, this technology has great potentiality to serve customers who have not been served before or who were not satisfied with their wired broadband technologies. The performance of BWA is limited by multipath fading, delay spread and co-channel interference [1].IEEE 802.16 standard and its associated industry consortium WiMAX forum promise to offer the users a high speed wireless service over a large area. The first version of the IEEE 802.16 standard operates in the range of $10-66 \mathrm{GHz}$ frequency and requires a Line-of-Sight (LOS) communication between the transmitter and the receiver.The later standards extended its operation through different PHY specifications to $211 \mathrm{GHz}$ frequency band enabling a Non Line-of-Sight (NLOS) communication. This later version uses a technique 
that can efficiently mitigate the performance impairments caused by the fading due to multipath signal propagation. Utilizing the advantages of Orthogonal Frequency Division Multiplexing (OFDM) technique the PHY layer is able to provide a robust broadband service in a hostile wireless channel environment.The HiperMAN standard and the OFDM based physical layer of IEEE 802.16 are nearly identical because OFDM based physical layer has been designed in close cooperation with the European telecommunications standard High Performance Metropolitan Area Network (HiperMAN). Both OFDM based physical layers comply with each other and result in the emergence of a global OFDM system.AllWiMAX forum certified products for BWA comply with the both of these standards.In this paper IEEE 802.16 OFDM physical layer has been implemented using MatLab in order to investigate the system performances. Stanford University Interim (SUI) channel has been used as the channel model. There have been numerous channel models proposed for wireless communication system design. These channel models can be broadly classified as (i) Indoor channel models, and (ii) Outdoor channel models [19-20]. UWV channel models, Saleh-Valenzeula channel model, and IEEE 802.11 channel model are some of the examples of indoor channel models. Whereas Filtered White Gaussian Noise (FWGN), Clarke-Gans model, Modified Frequency Domain FWGN model, Time Domain FWGN model, Jakes model, Ray based channel model, Frequency Selective channel model, and Stanford University Interim (SUI) channel models are the examples of outdoor channel models.In this investigation we chose SUI model because of its important characteristics. Some of the important characteristics of SUI channel are as follows: (a) it has a higher path loss as compared to super cell architecture, (b) it includes both macroscopic and microscopic fading effects, (c) it considers both co-channel and adjacent channel interference, and (d) it takes account of high multipath delay and Doppler spread [2]. The SUI channel also includesmany diversified parameters such as terrain, antenna specification, wind speed or traffic range, and bandwidth. Erceg path loss model has been used to represent a real world scenario. The $K$ factor is a very important parameter of this channel model, it is the ratio of power in the fixed component to the power in the variable component.The $K$ factor depends upon BTS and CPE heights, bandwidth, distance from the antenna, environmental condition including wind, traffic, and season. However, SUI channel has much more realistic approach as the $K$ factor for all these 6 channels namely SUI-1, SUI-2, SUI3, SUI-4, SUI-5, and SUI-6 are very different [2]. The rest of this paper is organized as follows: Section 2 briefly explains OFDM modulation technique. A comprehensive description of SUI channel has been presented in section 3. The simulation model and results have been presented in section 4 . This paper is concluded with section 5 .

\section{OFDM MODULATION}

The Orthogonal Frequency Division Multiplexing (OFDM) is a multi-carrier modulation (MCM). OFDMwas introduced to support a very high data rate. The main advantage of OFDM is that it can minimize the Inter Symbol Interference (ISI). It is also considered a spectrum efficient modulation scheme originated from its orthogonal spectrum utilization. Some of the other advantages of OFDM are: (a) it can cope with the multipath fading with less computational complexity, (b) it is more resistant to frequency selective flat fading, (c) it uses computationally efficient FFT and IFFT algorithm, (d) it is suitable for coherent demodulation, (e) it can ensure the required Quality of Service (QoS) to the end users depending on the bandwidth, and (f) it can assist diversity techniques (i.e., time diversity and frequency diversity). OFDM has some disadvantages too. Some of the disadvantages of OFDM are: (a) it requires a strong synchronization between the transmitter and the receiver, (b) it is very much sensitive to phase noise and frequency offset, (c) it is not power efficient because the Fast Fourier Transform (FFT) algorithm and Forward Error Correction (FEC)are always active, (d) it cannot take the advantages of the diversity gain if few sub-carriers are allotted to each user,and (e) it requires a high peak to average ration RF power amplifier to avoid amplitude noise.Like most multi-carrier modulation the main principle of OFDM is also to divide into several parallel bit streams which are used to 
modulate several sub-carriers. The subcarrier which has a guard band ensures the non overlap of each other. OFDM is a special form MCM technique, which employs densely spaced orthogonal sub-carriers and overlapping spectrums. OFDM does not require the uses of bandpass filters as the subcarrier is orthogonal in nature. So the bandwidth is very efficiently used without causing the Inter Carrier Interference (ICI). The bandwidth can be reduced by removing the guard band and allowing the subcarrier to overlap. It is still possible to recover the individual subcarrier though they overlapped in the spectrum provided,if theorthogonality condition is maintained. By performing Fast Fourier Transform (FFT) on the input bit stream the orthogonality is achieved.Because of the combination of multiple low data rate subcarriers, OFDM provides a high data rate with a long symbol duration. Depending on the channel coherence bandwidth it reduces or completely eliminates the effects of Inter Symbol Interference (ISI), which is a common phenomenon in any multipath channel environment with short symbol duration. The basic OFDM transmitter and receiver are illustrated in Fig. 1.

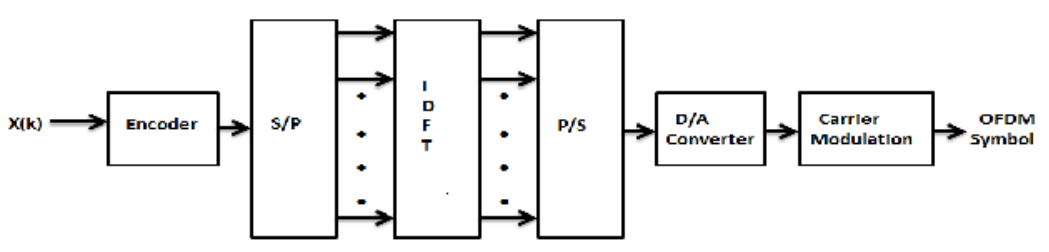

(a) OFDM Transmitter

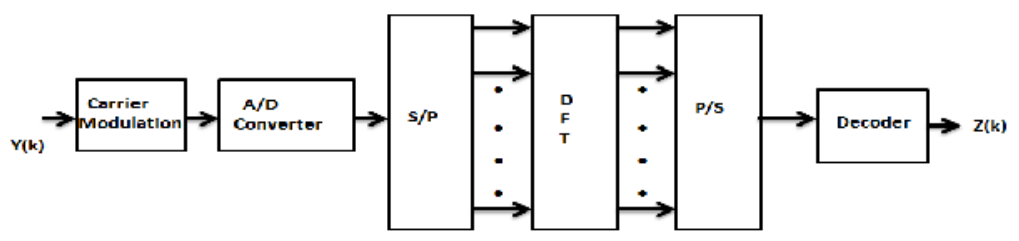

(b) OFDM Receiver

Fig. 1:(a) OFDM Transmitter and (b) OFDM receiver

In OFDM Discrete Fourier Transform (DFT) and Inverse Discrete Fourier Transform (IDFT) are used to implement the orthogonal signals. Since all the orthogonal sub-carriers have finite duration the spectrum of OFDM signal contains the summation of a set of sinc functions shifted in the frequency domain as shown in Fig.2. The orthogonality of the subcarriers of an OFDM system can be jeopardized when the same is sent through a multipath channel. A multipath channel introduces Inter Symbol Interference (ISI) and Inter Carrier Interference (ICI). Cyclic Prefix (CP) is added with the OFDM symbol to combat ISI and ICI. The CP contains a copy of the last part of the OFDM symbol which is attached to the front of transmitted OFDM symbol. The length of the CP must be longer than that of the maximum delay spread of the multipath environment. To take the advantages of the $\mathrm{CP}$ certain position within the $\mathrm{CP}$ is chosen as the sampling starting point denoted by $\mathrm{T}_{\mathrm{x}}$ at the receiver, which must satisfies a condition defined by $\tau_{\max }<T_{x}<T_{g}$, where $\tau_{\max }$ is the maximummultipath spread, $\mathrm{T}_{\mathrm{g}}$ is the duration of a CP. Once the above condition is satisfied, the ISI can be removed as the previous symbol will only have effect over samples withinthe range $\left[0, \tau_{\max }\right]$. The sampling period starting from $T_{x}$ will encompass the contribution from all the multipath components so that all the samples experience similar 


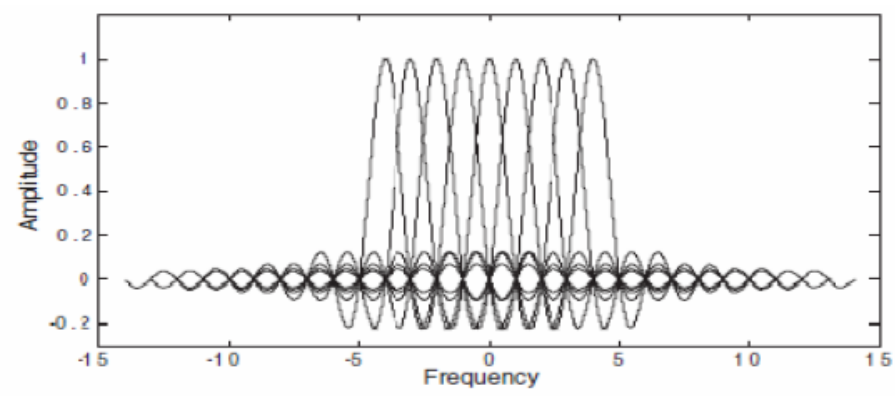

Fig.2: The spectrum of OFDM signal.

channel condition and there is no ICI.OFDM system was designed to decrease the data rate at the subcarrier level to satisfy the condition $T_{\mathrm{s}} \tau_{\max }$, where $\mathrm{T}_{\mathrm{s}}$ is the duration of the symbol. By using the Serial-to-Parallel (S/P) the symbol duration is increased(see Fig. 1) and hence the multipath effects are reduced effectively. The insertion of higher valued $\mathrm{CP}$ helps to combat multipath effects but at the same time it cancause higher loss of energy. Thus, a trade-off between these two parameters must be maintained to obtain a reasonable system performance.The performance of OFDM system is also limited by the constraints namely the availability of bandwidth, required data rate, tolerable delay spread, and Doppler shifts. Some of the basic design parameters of OFDM system are number of subcarriers, symbol duration and CP length, subcarrier spacing, modulation type per subcarrier, and FEC coding.

\section{The Sui Channel}

In order to investigate the performances of OFDM based BWA an accurate channel model needs to be considered. Usually all the wireless channels are characterized by path loss (including shadowing), multipath delay spread, fading characteristics, Doppler spread, and co-channel and adjacent channel interference.Ricean distribution can be used for characterization of narrow band received signal fading. In this distribution the key parameter is the $K$-factor, which is defined as the ratio of the "fixed" component power and the "scatter" component power. An empirical model was derived from a $1.9 \mathrm{GHz}$ experimental data set collected in a typical suburban environment for transmitter antenna heights of approximately $20 \mathrm{~m}$ [7]. The model presented in [7] is as follows:

$$
K=F_{s} F_{h} F_{b} K_{0} d \gamma u
$$

,where $F_{s}$ is a seasonal factor, $\mathrm{F}_{\mathrm{h}}$ is the receiving antenna height factor, $F_{b}$ is the beam width factor, $\mathrm{K}_{\mathrm{o}}$ and $\gamma$ are regression coefficients, $u$ is a lognormal variable which has mean at $0 \mathrm{~dB}$ and a standard deviation of $8.0 \mathrm{~dB}$. Some values of the parameters are $\mathrm{F}_{\mathrm{s}}$ as 1.0 in summer and 2.5 in winter respectively, $K_{o}$ and $\gamma$ are regression coefficients $\left(K_{o}=10 ; \gamma=-0.5\right)$. The receiving antenna height factor $\mathrm{F}_{\mathrm{h}}$ is defined by $\mathrm{F}_{\mathrm{h}}=0.46(\mathrm{~h} / 3)$, where $h$ is the receiving antenna height in meters, the beam width factor $\mathrm{F}_{\mathrm{b}}$ is defined by $\mathrm{F}_{\mathrm{b}}=(\mathrm{b} / 17)-0.62$, where $b$ is in degrees. The empirical model proposed in [7] has been confirmed by the experimental work presented in [8]. An independent set of experimental data was acquired in San Francisco Bay Area at $2.4 \mathrm{GHz}$ and with similar antenna heights mentioned in [7] which has been reported in [8].It has also been shown that the experimental data presented in [8] very closely matches with the model presented in [7].The narrow band $K$-factor distribution was found to be lognormalwith the median as a simple function of season, antenna height, antenna beam width, and distance. The standard deviation was found to be about $8 \mathrm{~dB}$. Using this model, it can be found that $K$-factor decreases as the distance from the antenna and the antenna beam width is increased. One of our focus is to determine $K$-factor that meets the requirement that $90 \%$ of all locations within a cell have to be served with $99.9 \%$ reliability. Calculating the $K$-Factor is quite complex as it involves path loss, delay spread, 
antenna correlation (if applicable), specific modem characteristics, and other parameters that influence system performance. However an approximated value can be calculated with much easier steps. First we select $90 \%$ of the users with the highest $K$-factors over the cell area. Secondly we can obtain the approximate value by selecting the minimum $K$-factor within the set. This value of $K$-factor can be close or equal to 0 . In Fig. 3 shows fading cumulative distribution functions (CDFs) for various $K$ factors. For example, for $K=0 \mathrm{~dB}$ (i.e., linear $K=1$ ) a $30 \mathrm{~dB}$ fade occurs, which is very similar to a Rayleigh fading case (i.e., linear $K=0$ ). The significance of these fade probabilities actually depends on the system design such as whether diversity is included and the quality of service (QoS) being offered etc.

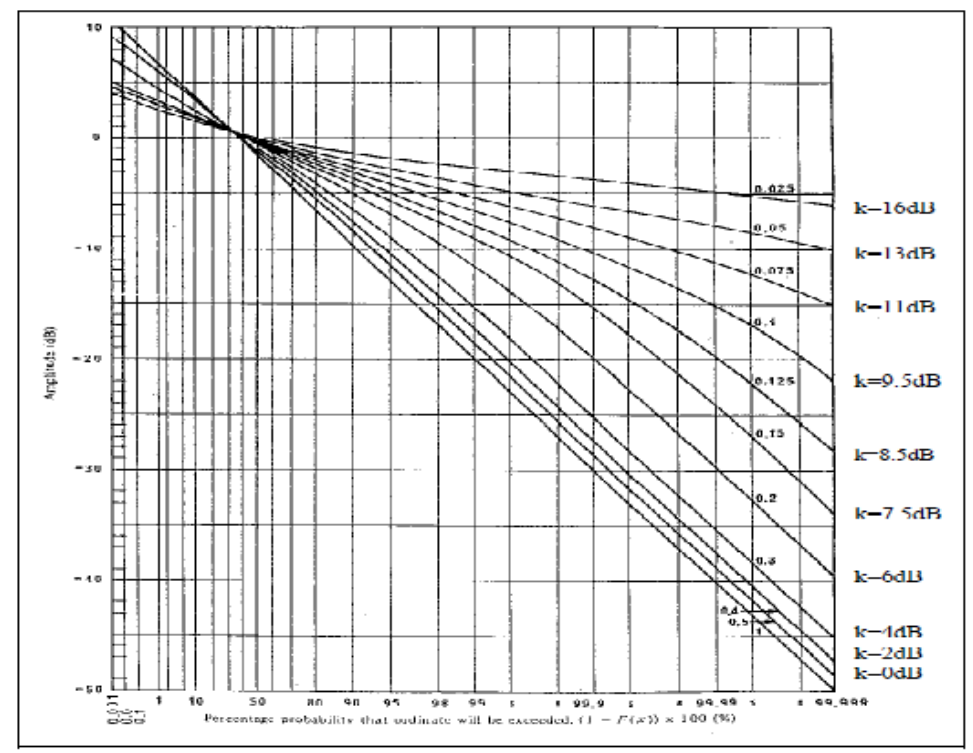

Fig. 3: Ricean fading distribution

When multiple antennas are used at the transmitter and/or at the receiver, the channel can be characterized by a matrix (i.e., Multi-Input-Multi-Output or MIMO in short) system, which is a natural extension of the developments in antenna array based communication system.

The advantages of multiple receive antennas, such as gain and spatial diversity, have been known and explained in some research works $[9,10,11]$, the use of transmit diversity has also been investigated in some recent works $[12,13]$. The advantages of MIMO communication, has been explained $[6,7,8,9,10]$. Channel models described above provide the basis for specifying channels for a certain given scenario. There are many possible combinations of parameters to obtain such channel descriptions. A set of 6 typical channels was selected for the three terrain types that are typical of the continental US [13]. In this section we have presented SUI channel models that we modified to account for $30^{\circ}$ directional antennas. These models can be used for simulation, design, development and testing of technologies suitable for fixed broadband wireless applications. The model parameters were selected based upon the statistical models. SUI channel models have 3 different types of terrain as shown in Table 1. Terrain C consisting of flat and light tree density so that the effects of the obstruction are low in this case. SUI-1 and SUI-2 are the channels taken into consideration to represent this type of terrain. The other two types of terrain models are B and A. The terrain B is characterized by flat/moderate tree density. SUI-3 and SUI4 have been proposed for this type of terrain. The terrain A is characterized by a hilly area which contains moderate to heavy tree density. SUI-5 and SUI-6 have been proposed for this type of 
terrain. The SUI channels can also be classified as two more categories namely SUI channel with low $K$-factor and SUI channel with high $K$-factor as shown in Table 2 and Table 3.

Table 1: Terrain of SUI model

\begin{tabular}{|l|l|l|}
\hline Terrain & Environmental Description & SUI Model \\
\hline C & Flat/Light Tree Density & SUI-1, SUI-2 \\
\hline B & Flat/Moderate Tree Density & SUI-3, SUI-4 \\
\hline A & Hilly/Moderate to Heavy Tree Density & SUI-5, SUI-6 \\
\hline
\end{tabular}

Table 2: SUI channel with low K-factor

\begin{tabular}{|c|c|c|c|}
\hline Doppler & Low Delay Spread & $\begin{array}{c}\text { Moderate Delay } \\
\text { Spread }\end{array}$ & High Delay Spread \\
\hline Low & SUI-3 & & SUI-5 \\
\hline High & & SUI-4 & SUI-6 \\
\hline
\end{tabular}

Table 3: SUI channel with high K-factor

\begin{tabular}{|c|c|c|c|}
\hline Doppler & Low Delay Spread & $\begin{array}{c}\text { Moderate Delay } \\
\text { Spread }\end{array}$ & High Delay Spread \\
\hline Low & SUI-1, SUI-2 & & SUI-5 \\
\hline High & & & \\
\hline
\end{tabular}

Table 2 and Table 3 depict that SUI-1 and SUI 2 have low delay spread because these two channels represent the terrain type $\mathrm{C}$ which is characterized by low tree density. Hence there are few obstructions in the signal propagation from a transmitter to a receiver.The other terrain types have moderate to high delay spread. All these contributing to the value of $K$ factor of the channel.

The general structure of SUI channel model is shown in Fig.4. This structure is generally used for MIMO channels and this includes other configurations such as Single Input Single Output (SISO) and Single Input Multiple Output (SIMO) as subsets. The structure is the same for the primary and interfering signals in case of SUI channel. The basic components of the SUI channel models are (a) input mixing matrix, (b) tapped delay line, and (c) output mixing matrix. The input mixing matrix

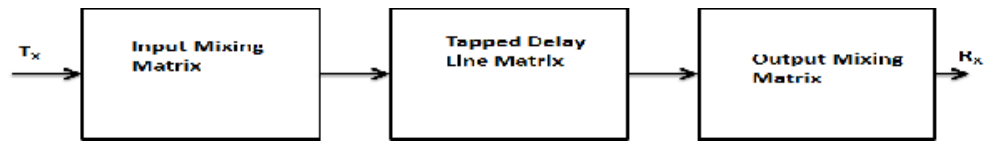

Fig. 4: Transmitter and receiver block diagram for SUI channel

modelsthe correlation between the input signals if multiple transmitting antennas are used. The tapped delay line matrixmodels the multipath fading of the channel.The multipath fading is modeled as a tapped-delay line with 3 taps all having different delays. The gain is associated with each tap which is characterized by a distribution (Ricean with a $K$-factor $>0$, or Rayleigh with $K$ factor $=0$ ) and the maximum Doppler frequency. The output mixing matrix models the correlation between the output signals in case we use multiple antennas.

Using the above general structure of the SUI Channel and thinking about the following scenario, six SUI channels are have been proposed for our experiment, which are representative of the real world channels. The following parameters (i) cell size:7 km, (ii) BTS antenna height: $30 \mathrm{~m}$,(iii 
)receive antenna height: $6 \mathrm{~m}$, (iv) BTS antenna beam width: $120^{\circ}$, (v) receive antenna beam width: omni-directional $\left(360^{\circ}\right)$ and directional $\left(30^{\circ}\right)$, (vi) verticalpolarization only, and (vii) $90 \%$ cell coverage with $99.9 \%$ reliability at each area covered.For a $30^{\circ}$ antenna beam width 2.3 times smaller RMS delay spread is used to compare to an omni-directional antenna [15]. Consequently, the second tap power is attenuated by an additional of $6 \mathrm{~dB}$ and the third tap power is attenuated by additional $12 \mathrm{~dB}$ (effect of antenna pattern and delay remain the same). For the omnidirectional receive antenna the tap delays and the powers are consistent [16].

Table 4: Characteristic of SUI-1 Channel

\begin{tabular}{|l|l|l|l|}
\hline Parameter & Tap 1 & Tap 2 & Tap 3 \\
\hline Delay ( S) & 0 & 0.4 & 0.9 \\
\hline $\begin{array}{l}\text { Power (Omni-directional Antenna) } \\
\text { (dB) }\end{array}$ & 0 & -15 & -20 \\
$90 \%$ K-factor (Omni-directional) & 4 & 0 & 0 \\
$75 \%$ K-factor(Omni-directional) & 20 & 0 & 0 \\
\hline $\begin{array}{l}\text { Power }\left(30^{\circ} \text { Antenna) }(\mathrm{dB})\right. \\
90 \% \text { K-factor }\left(30^{\circ}\right)\end{array}$ & 0 & -21 & -32 \\
$75 \%$ K-factor $\left(30^{\circ}\right)$ & 16 & 0 & 0 \\
\hline Doppler Shift $(\mathrm{Hz})$ & 72 & 0 & 0 \\
\hline
\end{tabular}

In Table 4 SUI-1 channel characteristics have been listed. In this type of channel model the terrain used is low in density hence the tap delay is low in this type of channel. There have been two types of antenna that are taken into account, firstly omni-directional and secondly a directional antenna with a tilt of $30^{\circ}$. In both the cases there are two different values of $K$ factor that have been considered. For this type of channel the antenna correlation is 0.7 , which results in the reduction of the channel capacity [18].The other parameters of SUI-1 channel are antenna correlation and $K$-factor. The value of antenna correlation under 0.5 has very negligible effect on the system capacity. The $K$-factor in this case is calculated and determined as 14 and 44.2 for $90 \%$ and $75 \%$ for directional antenna and for omni-directional respectively.In Table 5 shows the SUI-2 channel parameters. In compare to SUI-1 channel, SUI-2 channel has higher delay for Tap 3. The antenna correlation is less than that of SUI-1 channel. SUI-2 has an antenna correlation of 0.5 , which has almost no effect on the capacity of the model. The $K$-factor for omni-directional antenna is the same as SUI-1, but in case of directional antenna the K-factor reduces considerably.

Table 5: Characteristics of SUI-2 Channel

\begin{tabular}{|l|l|l|l|}
\hline Parameter & Tap 1 & Tap 2 & Tap 3 \\
\hline Delay ( S) & 0 & 0.4 & 1.1 \\
\hline Power (Omni-directional Antenna) (dB) & 0 & -12 & -15 \\
90\% K-factor (Omni-directional) & 2 & 0 & 0 \\
75\% K-factor(Omni-directional) & 11 & 0 & 0 \\
\hline Power $\left(30^{\circ}\right.$ Antenna) $(\mathrm{dB})$ & 0 & -18 & -27 \\
$90 \%$ K-factor $\left(30^{\circ}\right)$ & 8 & 0 & 0 \\
$75 \%$ K-factor $\left(30^{\circ}\right)$ & 36 & 0 & 0 \\
\hline Doppler Shift $(\mathrm{Hz})$ & 0.2 & 0.15 & 0.25 \\
\hline
\end{tabular}


Table 6: Characteristics of SUI-3 Channel

\begin{tabular}{|l|l|l|l|}
\hline Parameter & Tap 1 & Tap 2 & Tap 3 \\
\hline Delay ( S) & 0 & 0.4 & 0.9 \\
\hline Power (Omni-directional Antenna) $(\mathrm{dB})$ & 0 & -5 & -10 \\
90\% K-factor (Omni-directional) & 1 & 0 & 0 \\
75\% K-factor(Omni-directional) & 7 & 0 & 0 \\
\hline Power (30 Antenna) $(\mathrm{dB})$ & 0 & -11 & -22 \\
$90 \%$ K-factor $\left(30^{\circ}\right)$ & 3 & 0 & 0 \\
$75 \%$ K-factor(30 $)$ & 19 & 0 & 0 \\
\hline Doppler Shift $(\mathrm{Hz})$ & 0.4 & 0.3 & 0.5 \\
\hline
\end{tabular}

Table 6 shows the characteristic of SUI-3 which has a terrain type B. The tap delay in this case is more than that of the earlier mentioned channel models. The system is considered again with anOmni-directional and a directional antenna which have a $K$-factor of .5 and 1.6 for omnidirectional and 2.2 and 7 for directional for $90 \%$ and $75 \%$ respectively.

Table 7: Characteristic of SUI-4

\begin{tabular}{|l|l|l|l|}
\hline Parameter & Tap 1 & Tap 2 & Tap 3 \\
\hline Delay ( S) & 0 & 1.5 & 4 \\
\hline Power (Omni-directional Antenna) $(\mathrm{dB})$ & 0 & -4 & -8 \\
90\% K-factor (Omni-directional) & 0 & 0 & 0 \\
$75 \%$ K-factor(Omni-directional) & 1 & 0 & 0 \\
\hline Power $\left(30^{\circ}\right.$ Antenna) (dB) & 0 & -10 & -20 \\
$90 \%$ K-factor $\left(30^{\circ}\right)$ & 1 & 0 & 0 \\
$75 \%$ K-factor $\left(30^{\circ}\right)$ & 5 & 0 & 0 \\
\hline Doppler Shift $(\mathrm{Hz})$ & 0.2 & 0.15 & 0.25 \\
\hline
\end{tabular}

Table 8: Charactaristics of SUI-5

\begin{tabular}{|l|l|l|l|}
\hline Parameter & Tap 1 & Tap 2 & Tap 3 \\
\hline Delay ( S) & 0 & 4.0 & 10 \\
\hline Power (Omni-directional Antenna) & 0 & -5 & -10 \\
(dB) & 0 & 0 & 0 \\
90\% K-factor (Omni-directional) & 0 & 0 & 0 \\
$75 \%$ K-factor(Omni-directional) & 2 & 0 & 0 \\
$50 \%$ K-factor (omni-directional) & & & \\
\hline Power (30 Antenna) (dB) & 0 & -11 & -22 \\
$90 \%$ K-factor (30 $)$ & 0 & 0 & 0 \\
$75 \%$ K-factor(30 $)$ & 2 & 0 & 0 \\
$50 \%$ K-factor (30 $)$ & 7 & 0 & 0 \\
\hline Doppler Shift (Hz) & 0.2 & 0.15 & 0.25 \\
\hline
\end{tabular}

Table 7 characterizes SUI-4 channel model for type B terrain. SUI-4 has a further reduced antenna correlation but the delay is more in this model. In omni-directional the delay is $1.257 \mu \mathrm{s}$ and for the directional with a tilt of $30^{\circ}$ has a delay of $0.563 \mu$ s. The $K$-factor is further reduced in this channel model. The $K$-factors are 0.2 and 0.6 in omni-directional antenna. The $K$-factors are 1 and 3.2 for directional antenna. Table 8 and 9 show the characteristics of SUI-5 and SUI-6 channel models. In this model the antenna corellation is the same as the previous three models. But, the tap delay is less in SUI-5 as compared to SUI-6. The $K$ - factors for both SUI-5 and SUI-6 model are identical. 
Table 9: Charactaristics of SUI-6

\begin{tabular}{|l|l|l|l|}
\hline Parameter & Tap 1 & Tap 2 & Tap 3 \\
\hline Delay ( S) & 0 & 14.0 & 20 \\
\hline Power (Omni-directional Antenna) (dB) & 0 & -10 & -14 \\
90\% K-factor (Omni-directional) & 0 & 0 & 0 \\
75\% K-factor(Omni-directional) & 0 & 0 & 0 \\
$50 \%$ K-factor (omni-directional) & 1 & 0 & 0 \\
\hline Power (30 ${ }^{\circ}$ Antenna) (dB) & 0 & -16 & -26 \\
$90 \%$ K-factor $\left(30^{\circ}\right)$ & 0 & 0 & 0 \\
$75 \%$ K-factor $\left(30^{\circ}\right)$ & 2 & 0 & 0 \\
$50 \%$ K-factor $\left(30^{\circ}\right)$ & 5 & 0 & 0 \\
\hline Doppler Shift $(\mathrm{Hz})$ & 0.4 & 0.3 & 0.5 \\
\hline
\end{tabular}

\section{SUI SIMULATION MODEL AND RESULTS}

The simulation model mainly consists of three main components namely transmitter, receiver and channel. The channel used in the simulation is modeled as fading channel which will resemble the real scenario. This structure of the model simulated corresponds to the physical layer of the IEEE 802.16 (2004) Wireless MAN OFDM air interface. In this setup, we have just implemented the mandatory features specifications, while leaving the implementation of optional features for future work [17]. Fig. 5 (a) shows the architecture of a OFDM transmitter and Fig.5 (b) shows an OFDM receiver. In Fig. 5, the OFDM system treats the source symbols (e.g., the QPSK symbols) at the transmitter to be in the frequency domain. These symbols are used as the inputs to the IFFT block whichconverts the signal into the time domain. Each of the $N$ (i.e., $N$ is the number of subcarriers) input orthogonal sinusoids for the IFFT have a different frequency. The IFFT block modulates the $N$ sinusoids onto $N$ orthogonal subcarriers and provides an OFDM symbol. To overcome the overlapping of data cyclic prefix is inserted and then D/A converts it to analog one. After that this time-domain signal is transmitted across the channel. At the receiving end, receiving antenna receives an independent copy of the transmitted signal. Then the A/D converts that signal into digital one and cyclic prefix is removed as well. After passing through the FFT block the received signal transforms into the frequency domain. When we plotted the FFT output samples in the complex plain then it forms a constellation (such as QPSK). Before the transmission of data the cyclic prefix $(\mathrm{CP})$ is added to the data. This adding of $\mathrm{CP}$ helps in resisting against ISI and multipath propagation [16].IEEE 802.16 allows the insertion of cyclic prefix with various lengths such as $1 / 4,1 / 8,1 / 16$ and 1/32. The length of the cyclic prefix must be chosen which longer than the maximum delay spread of the target multipath environment. The transmitted data is then fed into the SUI channels for operation. At the receiver side the cyclic prefix is removed before doing any further processing of the signal.

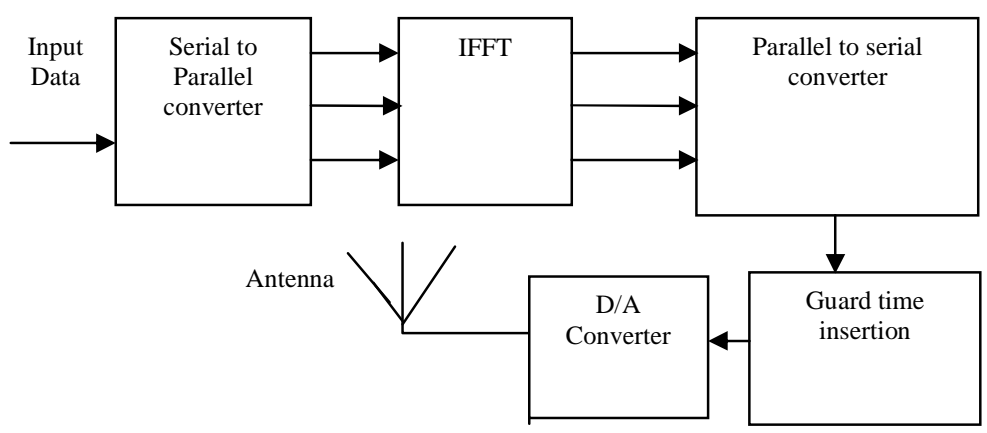

(a)Transmitter 


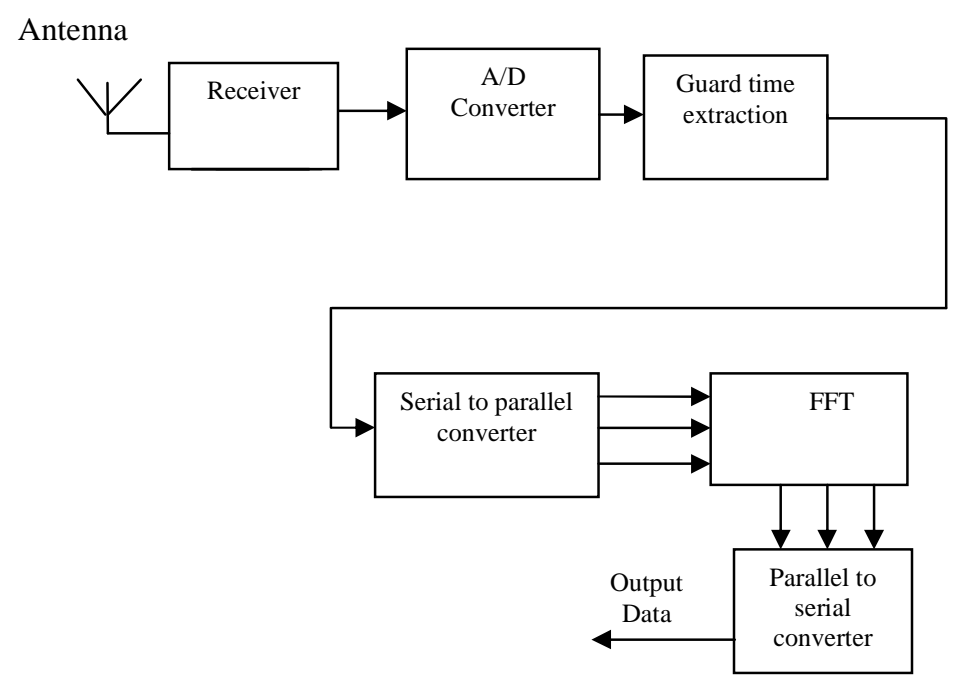

(b)Receiver

Fig.5: OFDM transmitter and receiver in SUI Channel

A modular have been developed using MatLab for our simulation work. The program contains the initialization parameters and input data. At the time of initialization are the number of simulated OFDM symbols, CP length, modulation, and the range of SNR values and SUI channel model for simulation can be set. In Table 10 some of the simulation parameters are listed. In Fig. 6 the flowchart of the program used has been shown. The input data stream are randomly generated where as the output variables are available in MatLab workspace while BER values for different SNR are stored in the text files which facilitate to draw plots. Each single block of the transmitter is tested with its counterpart of the receiver side to confirm each block is working perfectly.

Table 10: Simulation parameters

\begin{tabular}{|l|c|}
\hline \multicolumn{1}{|c|}{ Parameter } & Value \\
\hline FFT size. & 256 \\
\hline Number of used subcarriers. & 192 \\
\hline FFT Sampling frequency & $20 \mathrm{MHz}$ \\
\hline Ratio of Guard time to useful symbol time & $1 / 4,1 / 8,1 / 16,1 / 32$ \\
\hline
\end{tabular}




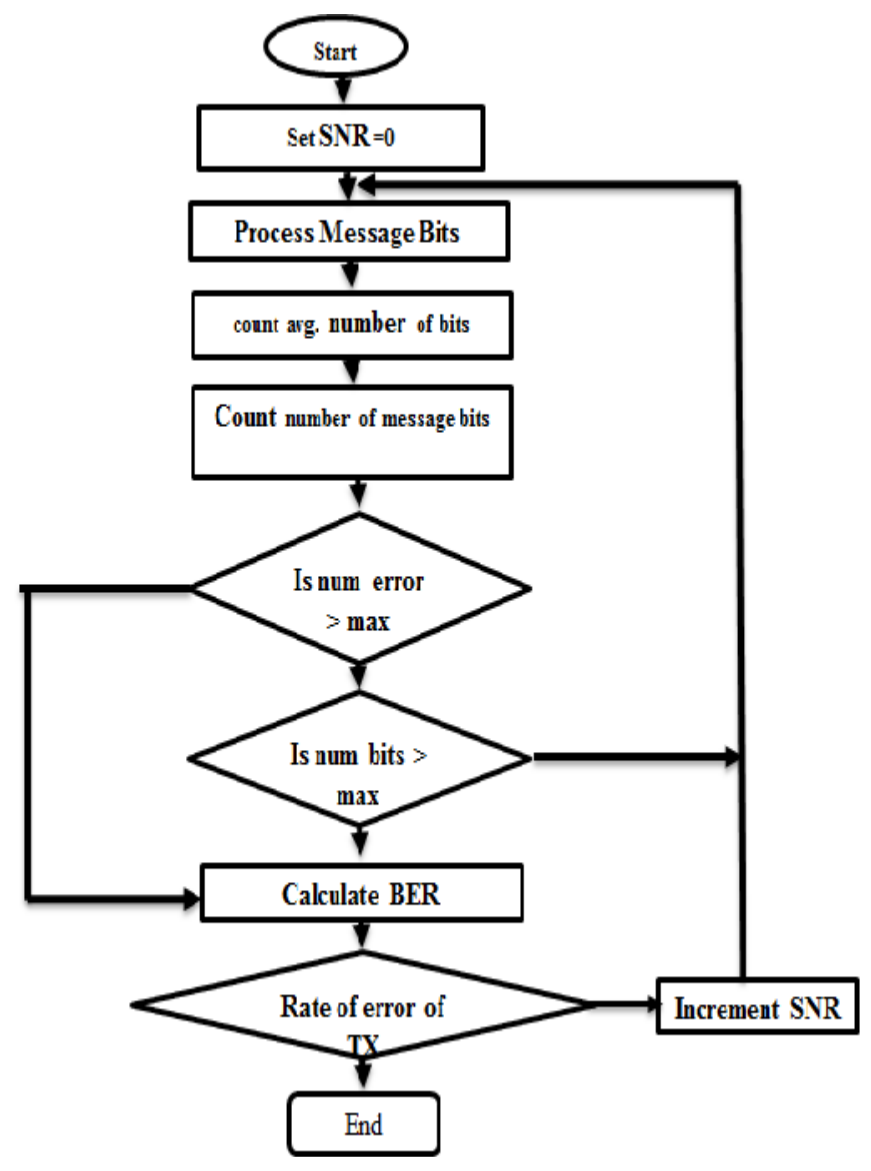

Fig. 6: Program flowchart

The simulation results of SUI-1 channel are shown in Fig. 7. In these simulations Binary Phase Shift Keying (BPSK) modulation was used and the cyclic prefix was varied. The theoretical result is also plotted in the same figure for comparison. Fig.7 shows that the BER decreases as the SNR $\left(\mathrm{E}_{\mathrm{b}} / \mathrm{N}\right)$ increases as usual. But, the cyclic Prefix plays an important role on the system performance. It is clearly shown that as the CP is increased for a given SNR the BER decreases for a given SNR. The simulation results for SUI-2 channel model are presented in Fig.8. The system performances are very similar to SUI- 1 channel model. The $\mathrm{CP}=1 / 4$ is the best choice for SUI-2 model. Unlike SUI-1 channel the other cyclic prefixes like 1/8, 1/16, and 1/32 perform almost in the same manner. For small SNR the performances are similar for 1/18, 1/16, and 1/32 CPs. But, the system performance is the best for $\mathrm{CP}=1 / 8$.

The simulations are repeated for SUI-3 channel and the results are shown in Fig. 9. This figure shows that the cyclic prefixes play no significant role on the system performances. Italso shows that the BER performance of the system are almost similar for $\mathrm{CP}=1 / 8,1 / 16$ and 1/32 although they have different effects for SUI-1 and SUI-2 channels. 
International Journal of Computer Networks \& Communications (IJCNC) Vol.5, No.2, March 2013

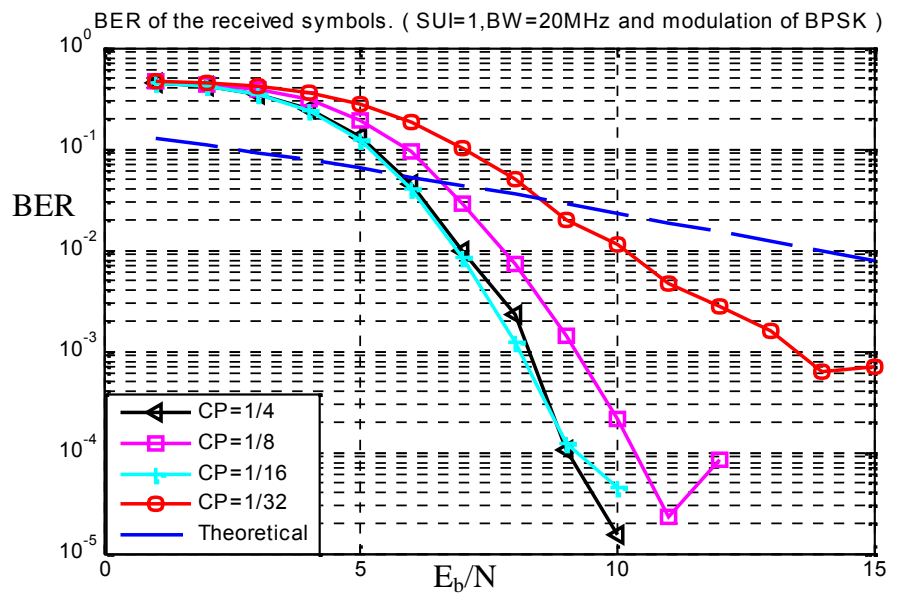

Fig. 7: Bit Error Rate of SUI-1 channel with BPSK

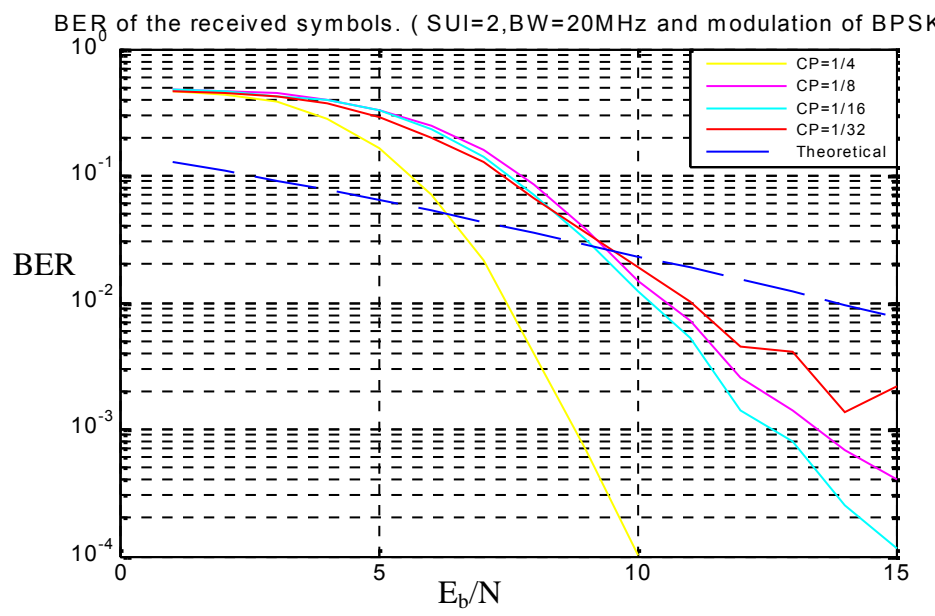

Fig.8: Bit Error Rate of SUI-2 channel with BPSK

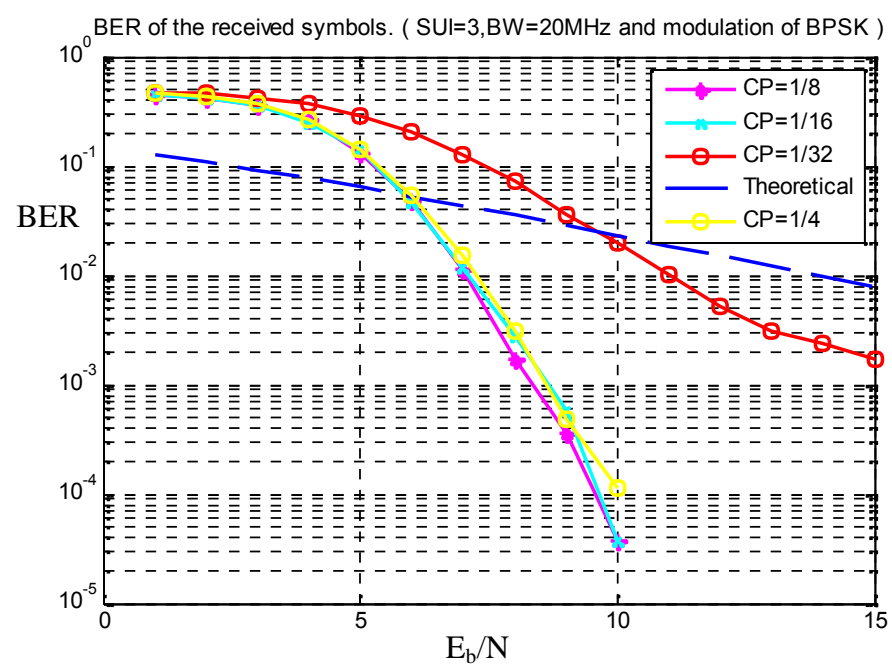

Fig.9: Bit Error Rate of SUI-3 channel with BPSK 
The BER rate performances for SUI-4 channel are shown in Fig. 10. It shows that the optimal CP for SUI-4 channel is $\mathrm{CP}=1 / 4$. The other most significant $\mathrm{CP}$ is $\mathrm{CP}=1 / 8$. The $\mathrm{CP}=1 / 16$ and $\mathrm{CP}=1 / 32$ have no effects on the BER at all. We also consider SUI-5 and SUI-6 in our simulations. The results are presented in Fig. 11 and Fig. 12. These figures show that the cyclic prefixes have no effects on the system performances. The perfect matching of the curves confirms our claims. In our investigation we also considered Quadrature Phase Shift Keying (QPSK) modulation. We conducted similar simulations as mentioned before but we changed the modulation to QPSK. The simulation results for QPSK with SUI-1 channel are presented in Fig. 13. The results show that $\mathrm{CP}=1 / 8$ is the best choice for $\mathrm{SUI}-1$ channel. The $\mathrm{CP}=1 / 16$ is a poor choice for SUI-1.

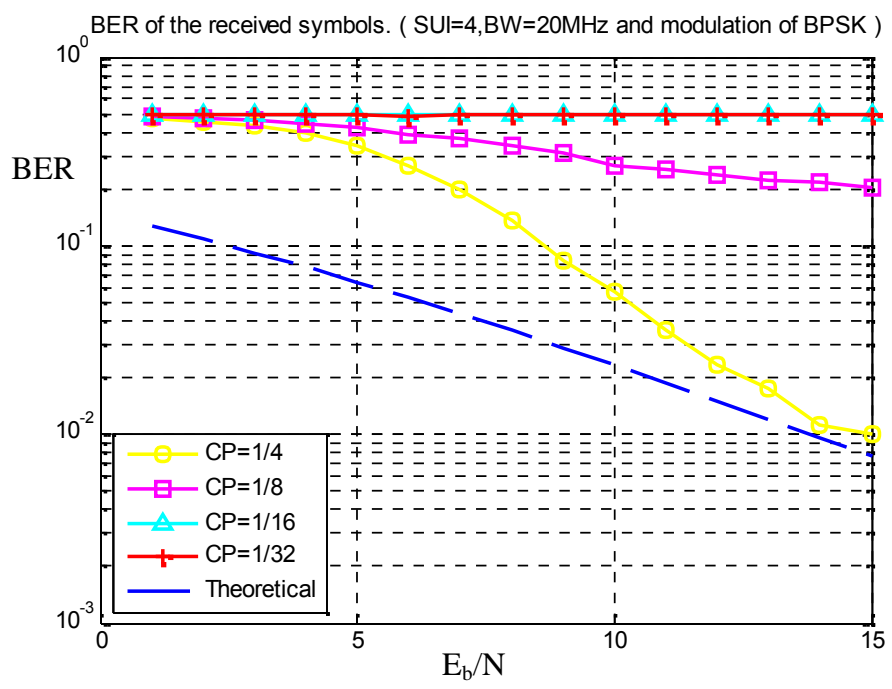

Fig.10: Bit Error Rate of SUI-4 channel with BPSK

The system performances of SUI-2 channels are shown in Fig. 14. This figure shows that CPs play important roles on the system performance. Different CPs has different impacts on the system performances. For example, it is shown that $\mathrm{CP}=1 / 8$ is the best choice for SUI- 2 channel. On the other than $\mathrm{CP}=1 / 32$ has little effect on the system performance.

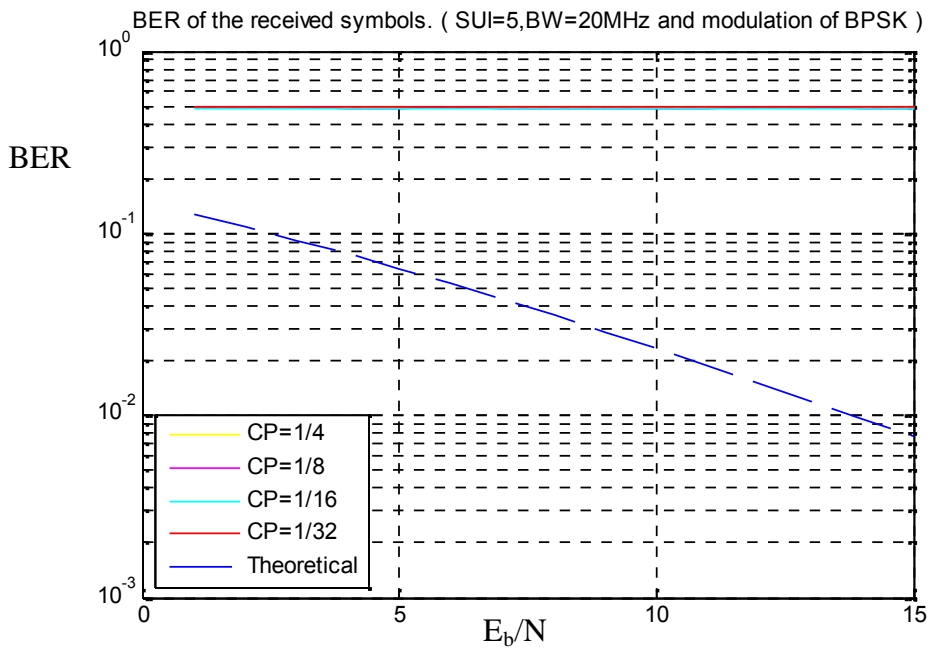

Fig.11: Bit Error Rate of SUI-5 channel with BPSK 
International Journal of Computer Networks \& Communications (IJCNC) Vol.5, No.2, March 2013

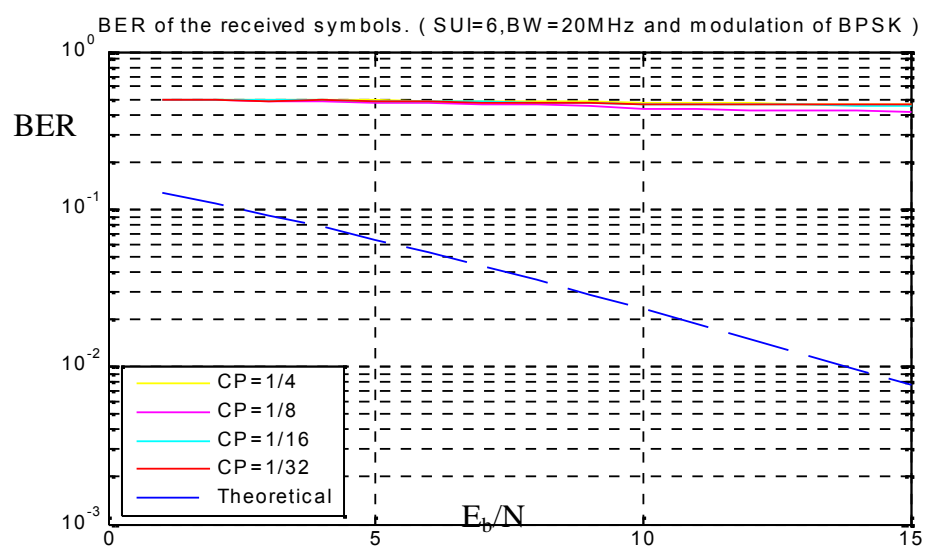

Fig.12: Bit Error Rate of SUI-6 channel with BPSK

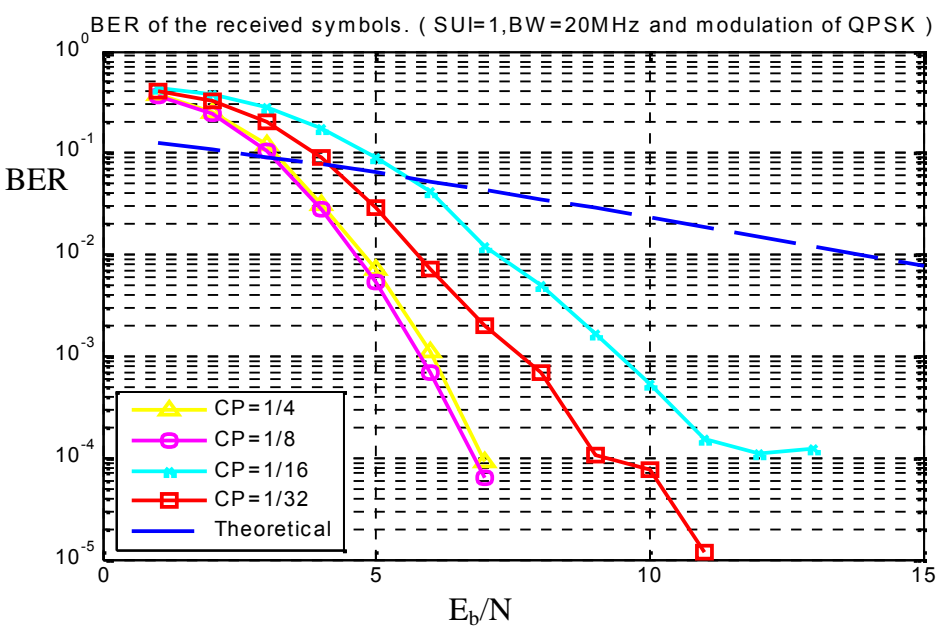

Fig.13: Bit Error Rate of SUI-1 channel with QPSK

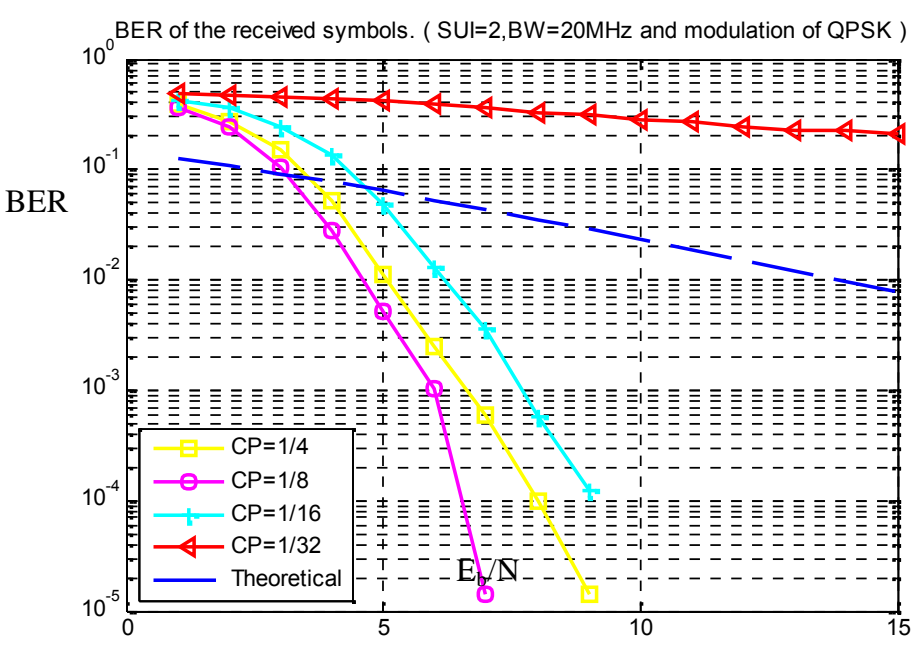

Fig.14: Bit Error Rate of SUI-2 channel with QPSK 


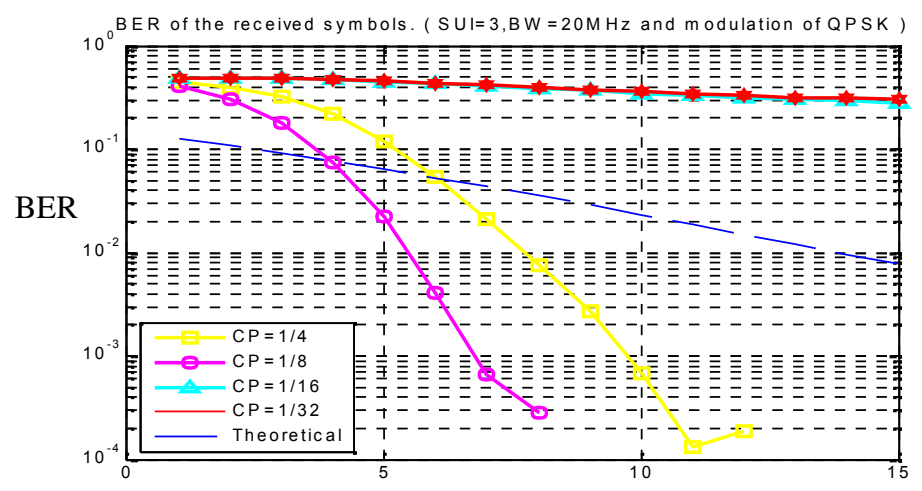

Fig.15: Bit Error Rate of EUI-3 channel with QPSK

The simulation results for SUI-3 channel are shown in Fig. 15. This figure shows that $\mathrm{CP}=1 / 32$ and $\mathrm{CP}=1 / 16$ have little effects on the system performance. But, $\mathrm{CP}=1 / 8$ is the best choice for SUI-3 model. The $\mathrm{CP}=1 / 4$ is also a good choice for SUI-3 channel.We also repeated the simulations for SUI-4, SUI-5 and SUI-6 channels. We found similar results like those of BPSK modulation as shown before. Hence, we did not repeat the results here. But, the major finding from those simulation results is that the CPshas little effect on SUI-4, SUI-5 and SUI-6 channels.

\section{Conclusions}

In this paper the performances of OFDM based IEEE 802.16d have been investigated. Different SUI channel models have been considered in this investigation. Some important parameters of OFDM like cyclic prefixes have been varied. Two modulation schemes namely BPSK and QPSK have been considered. The simulation results show that choosing an appropriate $\mathrm{CP}$ depends on the given conditions. Theoretically higher CP length should be chosen to cope with ISI and hence to improve system performance. But, in this investigation we show that $\mathrm{CP}$ should be chosen depending on the channel modes and the modulation schemes. For example, $\mathrm{CP}=1 / 4$ is the best choice for most the cases addressed in this paper. But, $\mathrm{CP}=1 / 8$ outperforms $\mathrm{CP}=1 / 4$ in some cases like when we used QPSK and SUI-3 channel model. So, the appropriate CP should be chosen depending on the channel and modulation used.

\section{REFERENCES}

[1] Hidehiro Matsuoka and Hiroki Shoki, (2003)“Comparison of pre-FFT and post-FFT processing adaptive arrays for OFDM systems in the presence of co-channel interference”, The 14th Proceedings of Personal, Indoor and Mobile Radio Communication, Vol.2, pp. 1603-1607

[2] www.Wirelessman.org

[3] William Stallings (2002), "Wireless: Communications and Networks", Pearson Education, pp. 139146

[4] LOS Versus NLOS available at http://www.conniq.com/WiMAX/nlos-los.htm

[5] K. Fazel, S. Kaiser, "Multi-Carrier and Spread Spectrum Systems: From OFDM and MC-CDMA to LTE and WiMAX", 2nd Edition, John Wiley \& Sons, 2008, ISBN 978-0-470-99821-2

[6] "Advantages and Disadvantages of OFDM available at http://sna.csie.ndhu.edu.tw/ cnyang/MCCDMA/tsld021.htm

[7] L.J. Greenstein, S. Ghassemzadeh, V.Erceg, and D.G. Michelson (1999), "Ricean K-factors in narrowband fixed wirelesschannels: Theory, experiments, and statistical models," In the Proceedings of Wireless Personal Multimedia Communication, May 21-23, Amsterdam, The Netherlands

[8] D.S. Baum et.al., (2000) "Measurements and characterization of broadband MIMO fixed wireless channels at $2.5 \mathrm{GHz}$ ", In the Proceedings of IEEE International Conference on Personal Wireless Communication, Hyderabad, December 2000.

[9] W.C. Jakes (1974), "Microwave Mobile Communications", John Wiley, New York, 1974. 
[10] R.A. Monzingo and T.W. Miller, (1980)“Introduction to Adaptive Arrays", JohnWiley, NewYork, 1980.

[11] K.W. Forsythe, D.W. Bliss, and C.M. Keller, (1999) "Multichannel Adaptive Beamforming and InterferenceMitigation in Multiuser CDMA Systems," In the proceedings of the thirty-third Asilomar Conf. on Signals, Systems \& Computers 1, Pacific Grove, Calif., 24-27 Oct. 1999, pp. 506-510.

[12] A. Wittneben, (1991) "Basestation Modulation Diversity for Digital SIMULCAST," In the proceedings of IEEE Vehicular Technology Conf., St. Louis, Mo., 19-22 May, pp. 848-853.

[13] V. Weerackody, (1993) "Diversity for Direct-Sequence Spread Spectrum Using Multiple Transmit Antennas," In the proceedings of IEEE International Communications Conference, Geneva, 23-26 May, pp. 1775-1779.

[14] T.L. Marzetta and B.M. Hochwald, (1999) "Capacity of a Mobile MultipleAntennaCommunicationLink inRayleigh Flat Fading," IEEE Transaction on Information Theory, Vol. 45, No. 1,1999, pp. 139-157.

[15] B.M. Hochwald and W. Sweldens, (2000) "Differential Unitary Space-Time Modulation," IEEE Transaction on CommunicationVol. 48 No. 12,pp. 45-48

[16] Simon Haykin (1988), "Digital Communication", John Wiley \& Sons, Inc

[17] IEEE std 802.16e 2005 and IEEE std 802.16 2004(Amendment and Corrigendum to IEEE std 802.16 2004), "IEEE standard for Local and metropolitan area networks part 16:Air Interface for Fixed and Mobile Broadband Wireless Access System Amendment 2:Physical and Medium Access Control Layers for Combined Fixed and Mobile Operation in Licensed Bands and Corrigendum", 1 February 2006.

[18] Syed M. Tabish Qaseem, and Adel A. Ali, (2005) "Effect of Antenna Correlation and Rician Fading on Capacity and Diversity Gains of Wireless MIMO Channels" In the proceedings of InternationalSymposium on Wireless Communication, Siena, Italy

[19] Theodore S. Rappaport, "Wireless Communication: Principles and Practices", Prentice Hall, Upper Saddle River, New Jersey, 2nd Edition

[20] Young Soo Cho and JaeKwan Kim, Won Young Yang and Chung Goo Kang, “ MIMO OFDM Wireless Communication in MatLab, IEEE Press and John Wiley

\section{AUTHORS}

Abdul HashibSiddiqueis agraduate student member of IEEE. He completed his B.Sc. Engineering degree from American International University-Bangladesh in 2008. He also completed his Master of Telecommunication(MTEL) degree from the same university in 2010. He has also completed his Ma ster of Science in Electrical Engineering from the Petroleum Institute, Abu Dhabi, United Arab Emirates in 2013. He had worked as a Lecturer in Electrical and Electronic Engineering department from 2009 to 2010 . He has more than 10 journal and conference papers to his credential.His research interests include Smart Power Grid, Distributed Generator, Wireless Power Transfer, Wireless Communication, and channel modeling.

A K M Arifuzzmanis a Lecturer at American International University- Bangladesh (AIUB) in EEE Department. He has been completed his B.Sc. Engineering degree in December 2010. He is a member of IEEE. He is actively involved in research activities in the field of Electrical Engineering. He conducted several investigations in this field. $\mathrm{He}$ has been published his research works in several journals. His research interests are DSP signal processing, wireless communication, nano-electronics, bio-sensor etc.

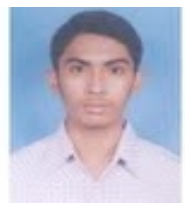

Mohammed Tarique completed his B.Sc. Engineering degree from Bangladesh University of Science and Technology (BUET) in 1992. He completed his MS degree from Lamar University, Texas, USA in 2001. He completed his PHD degree from the University of Windsor, Windsor, Canada in 2007. He has both industrial and academic experiences. Currently he is working with the Department of Electrical Engineering, Ajman University of Science and Technology (AUST), Fujairah, United Arab Emirates. He had also worked with the Department of Electrical and Electronic Engineering, American International University-

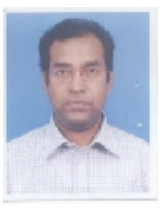
Bangladesh since 2007-2011. He supervised 7 Master students. His research works have been published in prestigious journal. He also presented his works in several conferences held in USA, Canada and Europe. His research interests are in Wireless Communication, Digital Signal Processing, Ad Hoc networks, Sensor Networks, and Antenna design. 